\title{
A breakthrough to explain sex bias?
}

Multiple sclerosis is an autoimmune disease of the central nervous system (CNS) that predominantly affects females but the reasons for this sex bias are unclear. Cruz-Orengo, Daniels et al. now report that increased expression of sphingosine-1phosphate receptor 2 (S1PR2) in the $\mathrm{CNS}$ may disrupt the integrity of the blood-brain barrier (BBB) and contribute to disease development.

Compared with their male counterparts, female mice on an SJL background are more susceptible to the development of experimental autoimmune encephalomyelitis (EAE), which is used as a model of multiple sclerosis. The authors used a whole-transcriptome microarray to identify sexually dimorphic genes in naive SJL mice and they found that $S 1 p r 2$ is differentially expressed in the female and male CNS. Additional expression analyses confirmed that both the mRNA and protein levels of S1PR2 are significantly increased in the cerebella of female SJL mice compared with in males. Signalling through S1PR2 regulates the integrity of the endothelial barrier and, in keeping with this, the authors found that vascular permeability was increased in the cerebella of female SJL mice compared with male mice. In addition, the induction of EAE in SJL mice further increased S1PR2 expression and vascular permeability in the cerebella and spinal cords of females but not in males. Notably, these sexual dimorphisms in S1PR2 expression and vascular permeability were not seen in C57BL/6 mice - a strain in which males and females show equal susceptibility to EAE.

To assess whether there are similar differences in humans, the authors examined post-mortem tissues from individuals with or without multiple sclerosis. Expression of S1PR2 was higher in the cerebella of individuals with multiple sclerosis compared with in those who did not have multiple sclerosis. Furthermore, females with multiple sclerosis showed increased expression of S1PR2 in the cerebellum compared with males with the

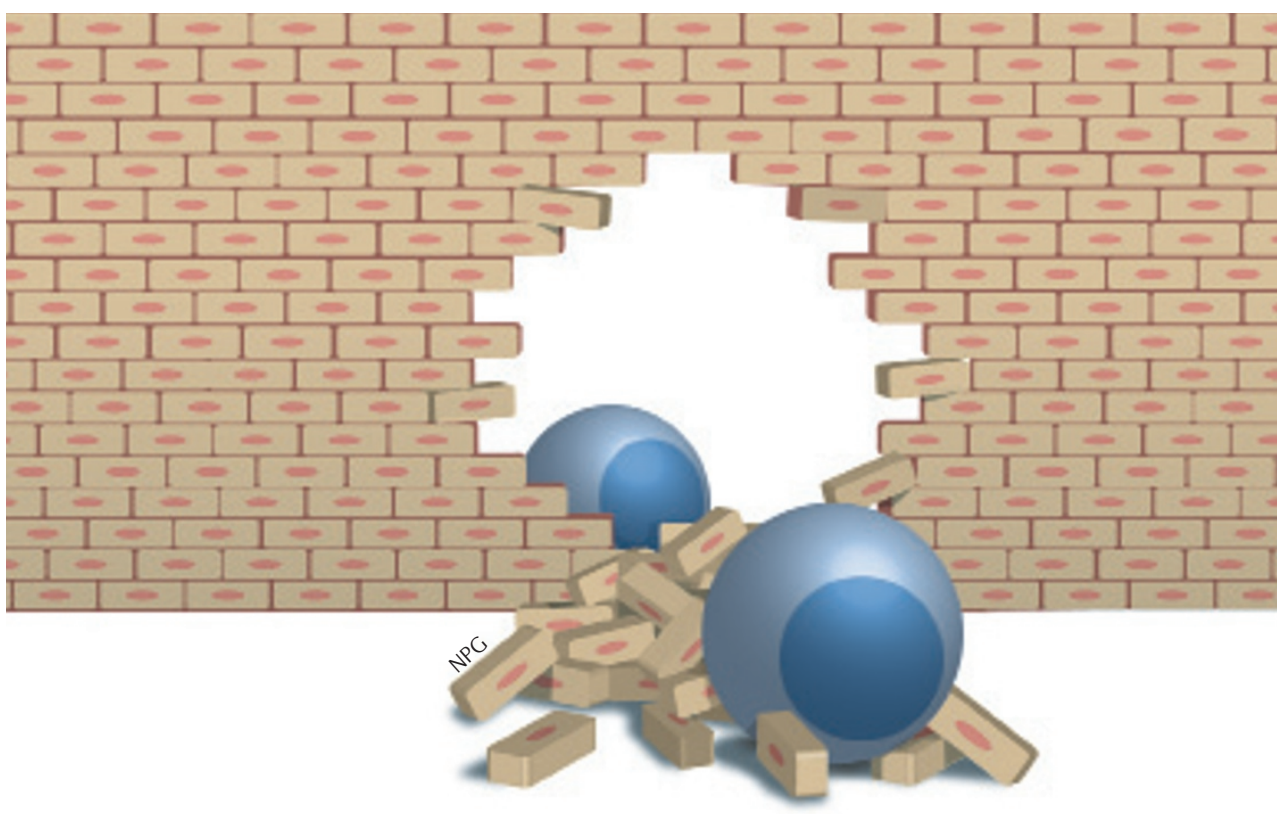

disease. The authors next examined whether increased S1PR2 expression contributes to the development of brain inflammation. They induced EAE in SJL mice and then treated the animals with JTE-013, which is a specific antagonist of S1PR2. Treatment with JTE-013 reduced disease scores in female SJL mice with EAE but had no effect on disease development in male SJL mice. S1PR2-deficient mice also showed decreased EAE severity compared with wild-type controls, which further suggests that S1PR2 signalling has a pathological role in demyelinating CNS disease.

Using an in vitro model of $\mathrm{BBB}$ function, the authors showed that signalling via S1PR2 disrupts the formation of adherens junctions between endothelial cells and leads to the loss of polarized expression of CXC-chemokine ligand 12 (CXCL12) at the abluminal surface of endothelial cells. Indeed, in vivo, they found that in male SJL mice and in both male and female C57BL/6 mice, CXCL12 was predominantly localized on the abluminal surfaces of the brain vasculature, whereas in female SJL mice, CXCL12 expression was primarily luminal. The authors conclude that enhanced S1PR2 signalling can contribute to the development of CNS inflammation by disrupting the BBB. They suggest that targeting S1PR2 could alleviate disease in patients with multiple sclerosis without impairing their general immune function.

Yvonne Bordon

ORIGINAL RESEARCH PAPER Cruz-Orengo, L. et al. Enhanced sphingosine-1-phosphate receptor 2 expression underlies female CNS autoimmunity susceptibility. J. Clin. Invest. http://dx.doi.org/10.1172//CI73408 (2014) 